\title{
Decline of SARS-CoV-2-specific IgG, IgM and IgA in convalescent COVID-19 patients within 100 days after hospital discharge
}

\author{
Huan $\mathrm{Ma}^{12 \dagger}$, Dan $\mathrm{Zhao}^{2 \dagger}$, Weihong Zeng ${ }^{12 \dagger}$, Yunru Yang ${ }^{2}$, Xiaowen $\mathrm{Hu}^{3}$, Peigen Zhou ${ }^{4}$, \\ Jianping Weng ${ }^{1,5,6}$, Linzhao Cheng ${ }^{7,8}$, Xueying Zheng ${ }^{1,5,6^{*}} \&$ Tengchuan Jin ${ }^{1,2,9^{*}}$ \\ ${ }^{1}$ The First Affiliated Hospital of USTC, Division of Life Sciences and Medicine, University of Science and Technology of China, Hefei 230001 , \\ China; \\ ${ }^{2}$ Hefei National Laboratory for Physical Sciences at Microscale, Laboratory of Structural Immunology, CAS Key Laboratory of Innate \\ Immunity and Chronic Disease, Division of Life Sciences and Medicine, University of Science and Technology of China, Hefei 230027, China; \\ ${ }^{3}$ Department of Pulmonary and Critical Care Medicine, the First Affiliated Hospital of USTC, Division of Life Sciences and Medicine, \\ University of Science and Technology of China, Hefei 230001, China; \\ ${ }^{4}$ Department of Statistics, University of Wisconsin-Madison, Madison, WI 53706, USA; \\ ${ }^{5}$ Joint Laboratory of Public Health, University of Science and Technology of China and Health Commission of Anhui Province, Hefei 230026, \\ China; \\ ${ }^{6}$ Clinical Research Hospital (Hefei) of Chinese Academy of Science, Hefei 230001, China; \\ ${ }^{7}$ Division of Life Sciences and Medicine, University of Science and Technology of China, Hefei Anhui 230026, China; \\ ${ }^{8}$ Johns Hopkins University School of Medicine, Baltimore, MD 21205, USA; \\ ${ }^{9}$ CAS Center for Excellence in Molecular Cell Science, Chinese Academy of Sciences, Shanghai 200031, China
}

Received July 13, 2020; accepted August 26, 2020; published online August 28, 2020

Citation: $\quad$ Ma, H., Zhao, D., Zeng, W., Yang, Y., Hu, X., Zhou, P., Weng, J., Cheng, L., Zheng, X., and Jin, T. (2021). Decline of SARS-CoV-2-specific IgG, IgM and IgA in convalescent COVID-19 patients within 100 days after hospital discharge. Sci China Life Sci 64, 482-485. https://doi.org/10.1007/s11427$020-1805-0$

Dear editor,

The 2019 novel coronavirus (later renamed as SARS-CoV-2 in February 2020) infected over 12 million people globally by early July 2020, causing mild to severe COVID-19 in millions. Monitoring the levels of antibodies such as immunoglobin (Ig) G, M and A that are specific to SARS-CoV2 and present in the blood provides not only an alternative method for diagnosing SARS-CoV-2 infection (including asymptomatic carriers), but also a simple way to monitor immune responses in convalescent patients or after vaccination. A high and persistent level of antibodies specific to SARS-CoV-2, especially those that can bind to and neutralize the virus, would be a strong indication that an im-

$\dagger$ Contributed equally to this work

*Corresponding authors (Tengchuan Jin, email: jint@ustc.edu.cn; Xueying Zheng, email: lxyzheng@ustc.edu.cn) munized host could resist to SRAS-CoV-2 infection. Currently, there are no effective drugs to specifically prevent or cure SARS-CoV-2 infection; therefore, host immune responses and antibody-based therapeutics will continue to play important roles in combating and later preventing COVID-19.

We previously established a set of diagnostic kits that quantitatively and sensitively measure the levels of serum IgG, IgM and IgA specific to the SARS-CoV-2 spike protein receptor binding domain (RBD), based on a cohort of 87 hospitalized COVID-19 patients and 483 negative controls (Ma et al., 2020). These antibodies that specifically bind to the RBD may block its interaction with a cell-surface protein ACE2 that serves as a main viral receptor. Previous studies demonstrated that the serum level of IgG that specifically binds to the RBD highly correlates with that of neutralizing antibody activity in blocking infection of SARS-CoV-2 or 
ACE2 targeting pseudo-viruses (Ni et al., 2020; Robbiani et al., 2020; Wu et al., 2020). Our RBD-specific, chemiluminescence-based kits are highly quantitative and sensitive for detecting SARS-CoV-2-elicited $\operatorname{IgA}, \operatorname{IgG}$ and $\operatorname{IgM}$ in the blood (Ma et al., 2020). During the optimal detection window of 16-25 days post illness onset, levels of RBD-specific IgA and IgG, but not IgM, were significantly higher in severe and moderate than mild COVID-19 patients (Ma et al., 2020).

To assess the levels of SARS-CoV-2-specific antibodies in COVID-19 convalescent patients over time after hospital discharge, we used the same kits for detecting RBD-specific $\operatorname{IgG}, \operatorname{IgM}$ and $\operatorname{IgA}$ levels in the blood of patients in this cohort and compared them to the levels during the hospitalization (Ma et al., 2020). Thirty-three convalescent patients living in Anhui province of China voluntarily came back to our clinic for revisit, 28-99 days after hospital discharge. Six of them were detected positive for SARS-CoV-2 nucleic acids and excluded in the current serology study. The information of 27 qualified convalescent patients is listed in Table S1 in Supporting Information, in the order of COVID19 severity during hospitalization. The table includes clinical information, discharge and revisit dates, and interval (28-99 days with a median 91 days) for each patient. The levels of the RBD-specific serum IgG, IgM and IgA (measured as relative light unit or RLU after 40 times' dilution) shortly before discharge and at revisit are tabulated in Table S2 in Supporting Information. In Figure $1 \mathrm{~A}-\mathrm{C}$, we plotted antibody levels soon before discharge and at revisit as cut-off index (COI), which is the ratio of RLU signal/cut-off value determined previously for serum IgG, IgM and IgA, respectively (Ma et al., 2020). Among the 27 convalescent patients, all (except \#10) who had severe or moderate COVID-19 symptoms and a high level of IgG during the hospitalization showed a significant reduction at revisit (Figure 1A). The remaining patients who had a low-level $\mathrm{IgG}$ during hospitalization stayed low at revisit. As expected, IgM levels in these convalescent patients reduced significantly or stayed low at revisit, except \#14 (Figure 1B). The RBD-specific IgA levels were also reduced significantly at revisit (Figure 1C), except for patient \#10, who also had an increased IgG, but not IgM. A few exceptional cases will need further studies.

We also attempted to estimate decline rates of virus-specific antibodies using a previously established exponential decay model of antibody kinetics after infection (Teunis et al., 2016). Based on the combined data of COI ratios before and after discharge for each of the 27 patients, we plotted decay curves for RBD-specific IgG, IgM and IgA over time (Figure 1D-F). The predicted days when convalescent patients' RBD-specific IgG reaches an undetectable level are approximately 273 days $(95 \%$ confidence interval ranging from 134-304 days or 4.5-10 months) after hospital dis- charge (Figure 1D), while the predicted decay time is 150 days and 108 days for IgM and IgA, respectively.

In summary, the initial data of this longitudinal study showed that the levels of SARS-CoV-2 RBD-specific antibodies in most COVID-19 convalescent patients reduced significantly or remained low within the first 100 days after discharge. Mathematical modeling and extrapolation predicts that the virus-specific IgG in this group of convalescent patients will disappear in 273 days ( $\sim 9$ months). Our data and analyses provide timely and critical information on how long the acquired humoral immune responses to this new coronavirus could persist. So far there are few papers studying the persistence of the SARS-CoV-2-elicited antibodies after recovery beyond two weeks (Long et al., 2020; Ni et al., 2020; Robbiani et al., 2020; Wu et al., 2020). In one study, blood samples (both cells and plasma) of six convalescent patients were collected two weeks after discharge and used to examine humoral and cellular immune responses (Ni et al., 2020). In another study (Wu et al., 2020), the serum $\mathrm{IgG}$ specific to the SARS-CoV-2 RBD and virus-neutralizing antibodies remained similarly low in 47 recovered patients two weeks after discharge. However, a recent study reported drastic declines of RBD-specific IgG and virus-neutralizing activities in 148 convalescent patients after an average of 39 days (Robbiani et al., 2020). The most recent study reported that $12.9 \%$ of the symptomatic group and $40 \%$ of the asymptomatic group became negative for IgG after 8 weeks, consistent with our findings of up to 99 days or 14 weeks.

Our current and other related studies lead to a conclusion that SARS-CoV-2 infection did not elicit a long-lasting humoral immune memory, similar to what has been reported with the SARS-CoV-1 (Cao et al., 2007). Our observation and decline kinetics modeling provide a guideline for SARS$\mathrm{CoV}-2$ vaccine designing as how to achieve long-lasting humoral immune response and memory. One way is to seek immunogens and adjuvants that elicit very strong immune responses such as virus-specific IgG induction, which can be easily monitored. For example, a recent clinical trial showed that an experimental vaccine using inactivated SARS-CoV-2 viruses with alum as the adjuvant only elicited comparable, but not much higher virus-specific IgG production than what we and others observed in hospitalized COVID-19 patients (Xia et al., 2020). Using more potent immunogens and adjuvants to enhance immune responses for stronger SARS$\mathrm{CoV}-2$ IgG production will be an important early indication for effective development of SARS-CoV-2 vaccines that are highly potent and long-lasting.

Although long-term data beyond 99 days after discharge are still in progress and our kinetics modeling needs confirmation, our current report provides timely information and fills the gap of knowledge for assessing the persistence of antibody levels in response to this novel coronavirus. The rapid reduction of antibodies (IgG, IgM and $\operatorname{IgA}$ ) specific to 

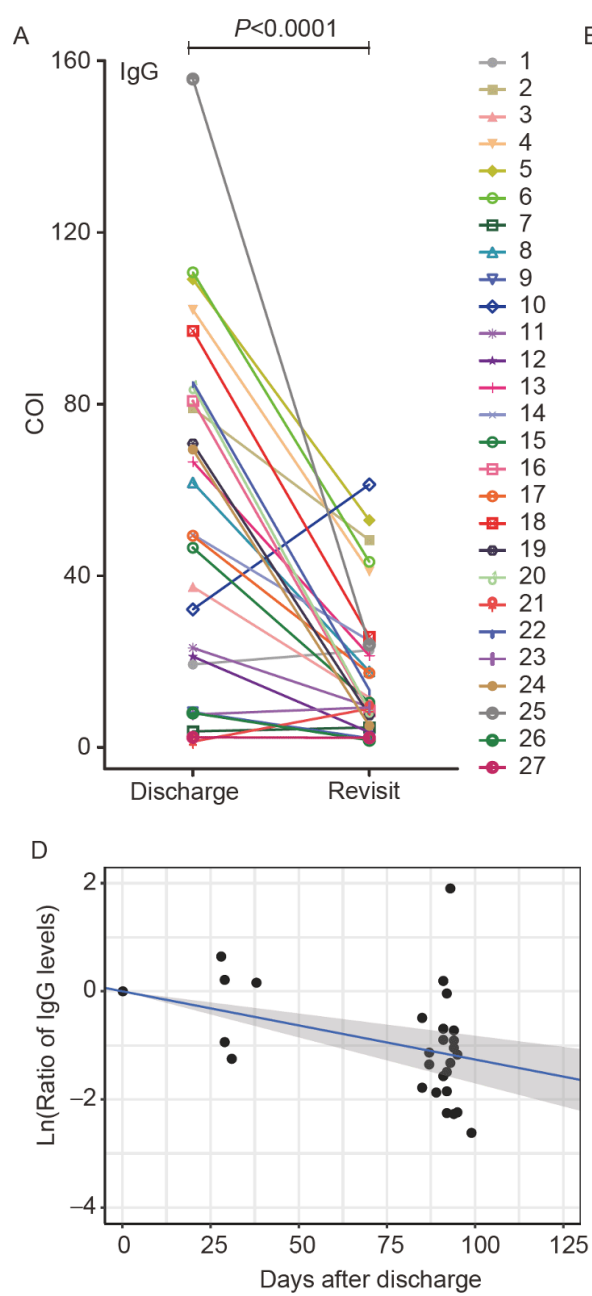
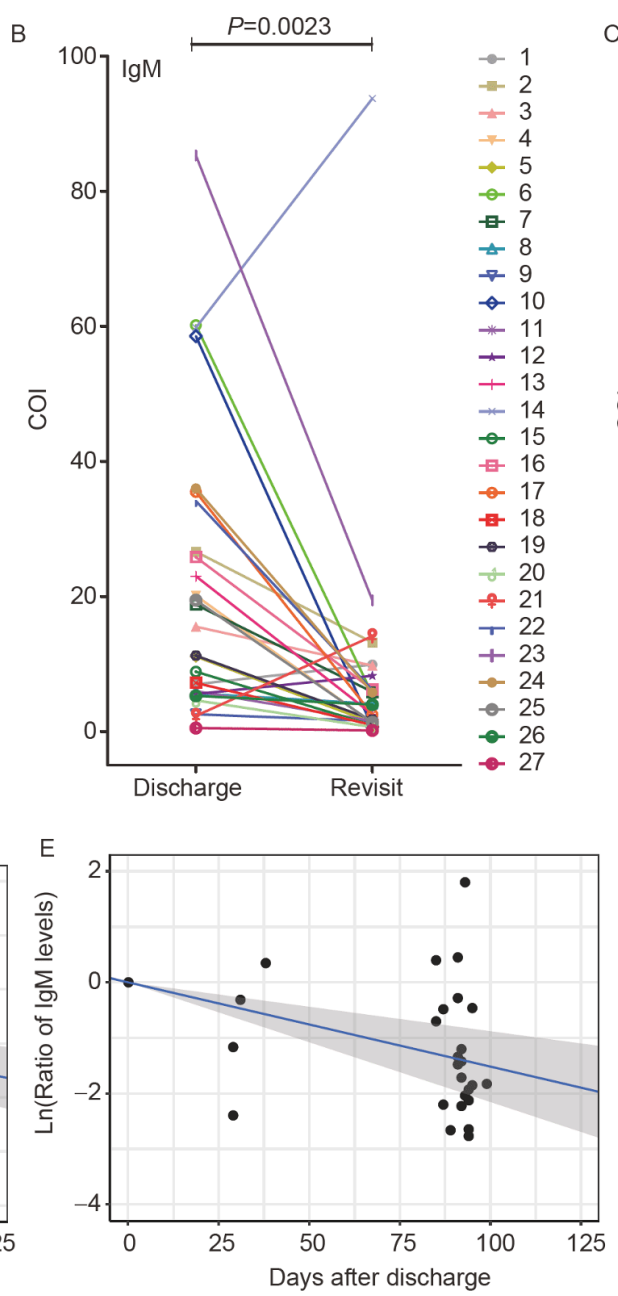
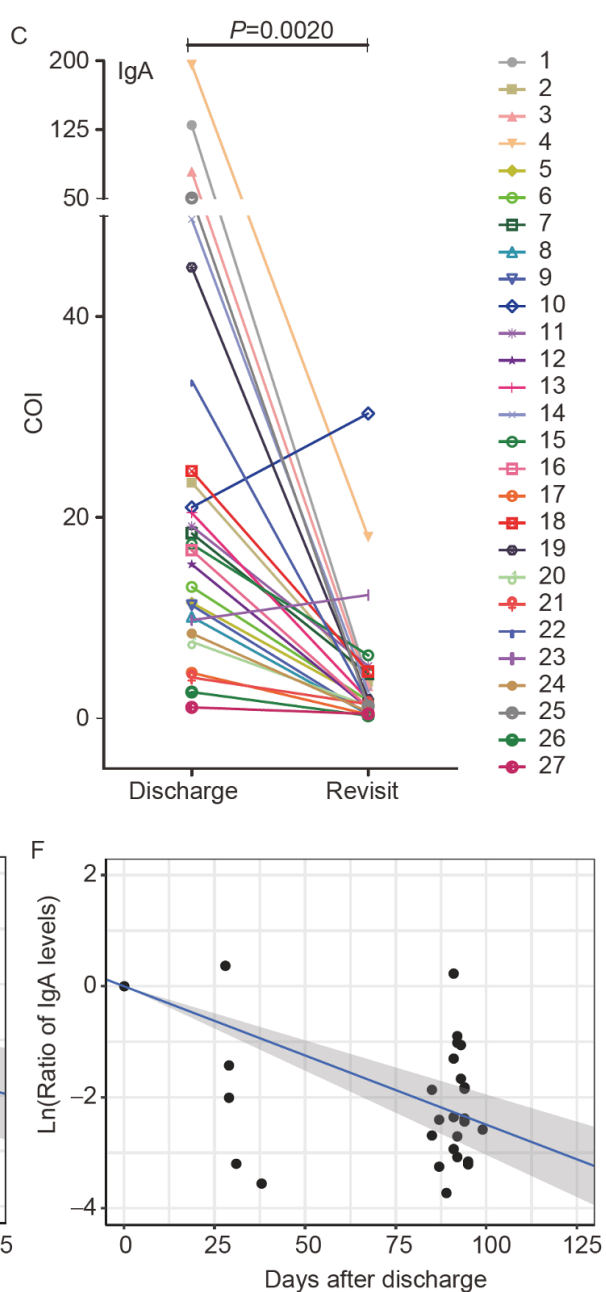

Figure 1 Changes of SARS-CoV-2 RBD-specific serum IgG, IgM and IgA levels in 27 convalescent patients near hospital discharge and at revisit $28-99$ days after discharge. A-C, The antibody levels are presented as cut-off index (COI), which is calculated as RLU signal divided by the cut-off value previously set for each of IgG, IgM and IgA, respectively. The $P$ values for the difference between discharge and revisit are $<0.0001,0.0023$ and 0.0020 for IgG, IgM and IgA, respectively. D-F, Decline curves for RBD-specific IgG (D), IgM (E) and IgA (F) over time, based on a mathematical model of exponential decay after its peak at recovery (soon before or at discharge). The ratios of COI at revisit versus discharge (day 0 ) is plotted by natural log scale for each patient's $\mathrm{IgG}$, IgM and IgA separately, as a function of time (days after discharge). See more details in supplemental Methods. The decay curve is marked as a blue line, and $95 \%$ confidence interval is marked as a grey zone for each type of SARS-CoV-2-specific antibodies.

SARS-CoV-2, which was observed in convalescent patients examined 4-14 weeks after discharge, warrants timely and close attention; however, our current data should be interpreted cautiously. First, we collected the data so far from a relatively small group of COVID-19 convalescent patients, who were chosen because we can trace changes of the virusspecific antibodies after discharge. Second, we measured only the antibodies specific to SARS-CoV-2 RBD in the study subjects. Third, we have not examined cellular immune responses as others did (Grifoni et al., 2020; Ni et al., 2020). Overall, our data are similar to what has been reported with SARS-CoV-1 infection: patients recovered from SARS had a rapid IgG decline that became undetectable after 3 years (Cao et al., 2007). However, a study reported the presence of long-lasting memory $\mathrm{T}$ cells reactive to the SARS-CoV-1 N protein in SARS patients recovered from
17 years ago (Le Bert et al., 2020). Nonetheless, this observational and longitudinal serology study provides timely and valuable information to aid current and future studies for addressing important issues such as how to use convalescent plasma or hyper-immunoglobins to treat COVID-19 patients and how to develop highly potent and long-lasting SARSCoV-2 vaccines.

Compliance and ethics The author(s) declare that they have no conflict of interest. This study was reviewed and approved by the Medical Ethical Committee of First Affiliated Hospital of USTC (approval number 2020-XG (H)-014 and 2020-XG(H)-009).

Acknowledgements We thank the staff and patients in The First Affiliated Hospital of USTC for their support in providing samples and clinical data collection. We would also like to thank Prof. Tian Xue and other colleagues in Division of Life Sciences and Medicine for their generous and profes- 
sional support. T.J. is supported by the Strategic Priority Research Program of the Chinese Academy of Sciences (XDB29030104), National Natural Science Foundation of China (31870731 and U1732109), the Fundamental Research Funds for the Central Universities (WK2070000108) and a COVID-19 special task grant supported by Chinese Academy of Sciences Clinical Research Hospital (Hefei) with Grant No. YD2070002017. M.H. is supported by the new medical science fund of University of Science and Technology of China (WK2070000130). J.W. and X.Y. are supported by the Fundamental Research Funds for the Central Universities with Grant Nos. YD9110004001 and YD9110002002, respectively.

\section{References}

Cao, W.C., Liu, W., Zhang, P.H., Zhang, F., and Richardus, J.H. (2007) Disappearance of antibodies to SARS-associated coronavirus after recovery. N Engl J Med 357, 1162-1163.

Grifoni, A., Weiskopf, D., Ramirez, S.I., Mateus, J., Dan, J.M., Moderbacher, C.R., Rawlings, S.A., Sutherland, A., Premkumar, L., Jadi, R.S., et al. (2020). Targets of T cell responses to SARS-CoV-2 coronavirus in humans with COVID-19 disease and unexposed individuals. Cell 181, 1489-1501.e15.

Le Bert, N., Tan, A.T., Kunasegaran, K., Tham, C.Y.L., Hafezi, M., Chia, A., Chng, M.H.Y., Lin, M., Tan, N., Linster, M., et al. (2020). SARSCoV-2-specific T cell immunity in cases of COVID-19 and SARS, and uninfected controls. Nature 584, 457-462.

Long, Q.X., Tang, X.J., Shi, Q.L., Li, Q., Deng, H.J., Yuan, J., Hu, J.L., Xu,
W., Zhang, Y., Lv, F.J., et al. (2020). Clinical and immunological assessment of asymptomatic SARS-CoV-2 infections. Nat Med 26, 1200-1204.

Ma, H., Zeng, W., He, H., Zhao, D., Jiang, D., Zhou, P., Cheng, L., Li, Y., Ma, X., and Jin, T. (2020). Serum IgA, IgM, and IgG responses in COVID-19. Cell Mol Immunol 17, 773-775.

Ni, L., Ye, F., Cheng, M.L., Feng, Y., Deng, Y.Q., Zhao, H., Wei, P., Ge, J., Gou, M., Li, X., et al. (2020). Detection of SARS-CoV-2-specific humoral and cellular immunity in COVID-19 convalescent individuals. Immunity 52, 971-977.e3.

Robbiani, D.F., Gaebler, C., Muecksch, F., Lorenzi, J.C.C., Wang, Z., Cho, A., Agudelo, M., Barnes, C.O., Gazumyan, A., Finkin, S., et al. (2020). Convergent antibody responses to SARS-CoV-2 in convalescent individuals. Nature 584, 437-442.

Teunis, P.F.M., van Eijkeren, J.C.H., de Graaf, W.F., Marinović, A.B., and Kretzschmar, M.E.E. (2016). Linking the seroresponse to infection to within-host heterogeneity in antibody production. Epidemics 16, 3339.

Wu, F., Wang, A., Liu, M., Wang, Q., Chen, J., Xia, S., Ling, Y., Zhang, Y., Xun, J., Lu, L., et al. (2020). Neutralizing antibody responses to SARSCoV-2 in a COVID-19 recovered patient cohort and their implications. medRxiv, https://doi.org/10.1101/2020.03.30.20047365.

Xia, S., Duan, K., Zhang, Y., Zhao, D., Zhang, H., Xie, Z., Li, X., Peng, C., Zhang, Y., Zhang, W., et al. (2020). Effect of an inactivated vaccine against SARS-CoV-2 on safety and immunogenicity outcomes. JAMA https://doi.org/10.1001/jama.2020.15543.

\section{SUPPORTING INFORMATION}

The supporting information is available online at https://doi.org/10.1007/s11427-020-1805-0. The supporting materials are published as submitted, without typesetting or editing. The responsibility for scientific accuracy and content remains entirely with the authors. 